\title{
STELLAR-WIND THEORY FOR O AND B STARS
}

\author{
PHILIP M. SOLOMON \\ Columbia University, New York, N.Y., U.S.A.
}

\begin{abstract}
The rocket-ultraviolet observations of strong Doppler-shifted absorption lines of Sirv, $\mathrm{Clv}, \mathrm{Nv}$ and other ions in the spectrum of $\mathrm{O}$ and $\mathrm{B}$ supergiants clearly indicate a high velocity outflow of matter from these stars. The presence of moderate ionisation stages in the stellar wind is conclusive evidence that the flow cannot be due to a high temperature corona as is the case for the solar wind. It is shown that the driving mechanism for the hot-star mass loss is radiation pressure exerted on the gas through absorption in resonance lines occurring at wavelengths near the maximum of the star's continuum flux. In the upper layers of these stars the outward force per gram of matter due to the radiation pressure can greatly exceed the gravitational acceleration making a static atmosphere impossible.

The problem of a steady-state moving reversing layer is formulated and the solution leads to predictions of mass-loss rates as a function of effective temperature and gravity for all hot stars. These results are in substantial agreement with the observations.
\end{abstract}

\section{Discussion}

Morton: This mechanism certainly must be an important cause of mass ejection in the hot supergiants. However, there may be additional effects such as convective or turbulent motions in the photosphere which could contribute to the rate of mass loss.

Solomon: Any additional mechanism would require very high temperatures in the flow $\left(T>10^{7} \mathrm{~K}\right)$ to account for the high velocities. These temperatures are clearly not present since no CIII, CIV, Si III or Sirv could possibly exist at $10^{7} \mathrm{~K}$, where the ions would be completely stripped with only one electron remaining.

Herbig: I would think that there might be two possible, directly observable consequences of such high-velocity mass outflow:

(a) Either the region within a cluster such as NGC 6231 (which contains the three actively ejecting stars mentioned by Hutchings) would be scoured clean of interstellar matter, leaving a cavity. Is there any sign of this? Or,

(b) In the other extreme, this volume would contain a great deal of highly excited material that might be detected directly. Or to put this last in another way, is direct detection possible of this $1000-3000 \mathrm{~km} \mathrm{sec}^{-1}$ material after it moves away from the immediate vicinity of these stars?

Solomon: The pressure in the flow would equal the interstellar pressure in an Hil region at about 1 psc from the star. The most interesting region might be the interface between the normal $\mathrm{HII}_{\mathrm{II}}$ region and the high velocity outflow. This might even yield detectable $\mathrm{X}$-rays.

Hearn: How is the ionization balance determined in calculating the radiation pressure? Have the transient effects of the ionization balance been included? What is the distribution of the electron temperature and density in the outer layers?

Solomon: The ionization equilibrium is determined by assuming a steady state between radiative ionization and recombination. This assumption is valid in the subsonic branch of the flow, where the time-scale for recombination is much less than the flow time-scale (time for the matter to move to a region where the density is lower by a factor of $e$ ). The transient effects are certainly important in the supersonic flow and this has been accounted for by actually summing up all recombinations and ionizations in the flow for each point in the model. The net effect is, that after velocities $>100 \mathrm{~km} \mathrm{sec}^{-1}$ are reached, the ionization remains almost constant since the time-scale for the flow becomes much less than the ionization time-scale.

Deutsch: Although you assert that your solution pertains only to the subsonic part of the flow, the line profile you have shown surely includes the absorption of the supersonic part. Can you say approximately how the different levels of supersonic flow are weighted in contributing to the line profile? 
Solomon: The model gives the number of ions as a function of the velocity of outflow. The optical depth at a velocity $V$ is inversely proportional to $\mathrm{d} V / \mathrm{d} r$, which is itself a function of $V$ and is determined by the model.

I should emphasize that while we regard the subsonic flow and the mass loss rates as accurate, the supersonic flow shown here is only presented to demonstrate that line profiles not unlike those observed can be obtained form the mass-loss rates derived from the subsonic flow.

Underhill: I have a wicked postulate I should like you to comment on. Suppose that a star had lost most of its outer envelope so that it was nearly peeled down to its helium-rich core and thus had an enriched $\mathrm{He} / \mathrm{H}$ content in its atmosphere, what do you think would happen to an expanding atmosphere generated in the manner you have suggested? Would the presumably hot He core contribute more momentum to the envelope by means of high-energy particles and strong UV radiation than reaches the outer atmosphere, when the core is enveloped in the normal deep radiative outer layers of a star?

Solomon: This postulate is too wicked for me to answer immediately. However, I expect that the star might adjust itself and that the He rich core would not be exposed. An enriched He atmosphere would probably not greatly effect the problem.

Praderie: (1) Do you include all resonance lines over the whole spectrum in the computation of the radiation pressure gravity term, and does this term vary with depth?

(2) A work by Bisnovetij-Kogan and Zeldovich considers the same type of hydrodynamical mass flow as Solomon, but the radiation pressure is that acting through the continuous spectrum; it is supposed that a sudden increase of the continuous absorption coefficient occurs near the surface of the star. Unhappily I cannot quote the values they find for the mass loss.

Solomon: We include all resonance lines at $\lambda>912 \AA$ of $\mathrm{C}, \mathrm{N}$, and $\mathrm{Si}$ ions and it is the increase of this term outwards that leads to the flow. This increase is not postulated but results from the model atmosphere. 